\title{
Al'tshuler-Aronov correction to the conductivity of a large metallic square network
}

\author{
Christophe Texier ${ }^{1,2}$ and Gilles Montambaux ${ }^{2}$ \\ ${ }^{1}$ Laboratoire de Physique Théorique et Modèles Statistiques, \\ UMR 8626 du CNRS, Université Paris-Sud, F-91405 Orsay Cedex, France. \\ ${ }^{2}$ Laboratoire de Physique des Solides, UMR 8502 du CNRS, \\ Université Paris-Sud, F-91405 Orsay Cedex, France.
}

(Dated: April 5, 2007)

\begin{abstract}
We consider the correction $\Delta \sigma_{\text {ee }}$ due to electron-electron interaction to the conductivity of a weakly disordered metal (Al'tshuler-Aronov correction). The correction is related to the spectral determinant of the Laplace operator. The case of a large square metallic network is considered. The variation of $\Delta \sigma_{\mathrm{ee}}\left(L_{T}\right)$ as a function of the thermal length $L_{T}$ is found very similar to the variation of the weak localization $\Delta \sigma_{\mathrm{WL}}\left(L_{\varphi}\right)$ as a function of the phase coherence length. Our result for $\Delta \sigma_{\text {ee }}$ interpolates between the known $1 \mathrm{~d}$ and $2 \mathrm{~d}$ results, but the interaction parameter entering the expression of $\Delta \sigma_{\text {ee }}$ keeps a $1 \mathrm{~d}$ behaviour. Quite surprisingly, the result is very close to the $2 \mathrm{~d}$ logarithmic behaviour already for $L_{T} \sim a / 2$, where $a$ is the lattice parameter.
\end{abstract}

PACS numbers: 73.23.-b ; 73.20.Fz ; 72.15.Rn

\section{INTRODUCTION}

At low temperature, the classical (Drude) conductivity of a weakly disordered metal is affected by two kinds of quantum corrections : the first one is the weak localization (WL) correction, a phase coherent contribution that originates from quantum interferences between reversed electronic trajectories. This contribution to the averaged conductivity depends on the phase coherence length $L_{\varphi}$ and the magnetic field : $\Delta \sigma_{\mathrm{WL}}\left(\mathcal{B}, L_{\varphi}\right)$. The temperature manifests itself through $L_{\varphi}$, since phase breaking may depend on temperature, e.g. if it originates from electron-electron ${ }^{\underline{1}}$ or electronphonon ${ }^{2}$ interaction.

In a metal, an electron is not only elastically scattered on the disordered potential, but, due to the electron-electron interaction, is also scattered by the electrostatic potential created by the other electrons. At low temperatures, when the elastic scattering rate $\left(1 / \tau_{e}\right)$ dominates the electronelectron scattering rate $\left(1 / \tau_{\mathrm{ee}}(T)\right)$, the motion of the electron is diffusive between scattering events with other electrons. In this regime, electronelectron interaction is responsible for a small depletion of the density of states at Fermi energy (called the DoS anomaly or the Coulomb dip) and a correction to the averaged conductivity as well, the socalled Al'tshuler-Aronov (AA) correction-3,4,5,6,7,8,9 (see Refs ${ }^{10,11,12}$ for a recent discussion). AA and WL corrections are of the same order (but this latter vanishes in a magnetic field). However, contrary to the WL, the AA correction is not sensitive to phase coherence and involves another important length scale : the thermal length $L_{T}=\sqrt{D / T}$ $\left(\hbar=k_{B}=1\right)$. The AA correction, denoted below $\Delta \sigma_{\text {ee }}\left(L_{T}\right)$, has been measured in metallic wires in several experiments $14,15,16,17$. From the exper- imental point of view, AA correction allows to study interaction effects in weakly disordered metals, but also furnishes a local probe of temperature in order to control Joule heating effects $\frac{15,17}{17}$, which is crucial in a phase coherent experiment.

All the works aforementioned refer to the quasione-dimensional (wire) or two-dimensional (plane) situations. Quantum transport has also been studied in more complex geometries like networks of quasi-1d wires. For example several studies of WL have been provided on large regular networks in honeycomb and square metallic networks ${ }^{18.19}$, in square networks etched in a $2 \mathrm{DEG}^{20}$, and in square and dice silver networks 21 . Theoretical studies of WL on networks have been initiated by the works of Douçot \& Rammal22,23 and improved by Pascaud \& Montambaux ${ }^{24}$ who introduced a powerful tool25 : the spectral determinant of the Laplace operator, that will be used in the following (see also Ref. $\left.\frac{26}{2}\right)$.

The aim of this paper is to study how the AA correction can be computed in networks. In a first part we briefly recall how the spectral determinant can be used to compute the WL. Then in a second part we will consider the AA correction.

\section{SPECTRAL DETERMINANT AND WEAK LOCALIZATION}

Interferences of reversed electronic trajectories are encoded in the Cooperon, solution of a diffusion-like equation $\left(\partial_{t}-D[\nabla-\right.$ 2ie $\left.A(x)]^{2}\right) \mathcal{P}_{c}\left(x, x^{\prime} ; t\right)=\delta\left(x-x^{\prime}\right) \delta(t)$, where $A(x)$ is the vector potential. On large regular networks, when it is justified to integrate uniformly the Cooperon over the network (see Ref ${ }^{27}$ for a discussion of this point) it is meaningful to introduce the space-averaged Cooperon $\mathcal{P}_{c}(t)=\int \frac{\mathrm{d} x}{\mathrm{Vol}} \mathcal{P}_{c}(x, x ; t)$ 
then

$$
\begin{aligned}
\Delta \sigma_{\mathrm{WL}} & =-\frac{2 e^{2} D}{\pi} \int_{0}^{\infty} \mathrm{d} t \mathrm{e}^{-t / \tau_{\varphi}} \mathcal{P}_{c}(t) \\
& =-\frac{2 e^{2}}{\pi} \frac{1}{\operatorname{Vol}} \frac{\partial}{\partial \gamma} \ln S(\gamma)
\end{aligned}
$$

where $\tau_{\varphi}=L_{\varphi}^{2} / D$ is the phase coherence time. The factor 2 stands for spin degeneracy. We have omitted in (12) a factor $1 / s$ where $s$ is the cross-section of the wires. The parameter $\gamma$ is related to the phase coherence length $\gamma=1 / L_{\varphi}^{2}$ (note that description of the decoherence due to electron-electron interaction in networks requires a more refined discussion 28,29 ). The spectral determinant of the Laplace operator is formally defined as $S(\gamma)=\operatorname{det}(\gamma-\Delta)=\prod_{n}\left(\gamma+E_{n}\right)$ where $\left\{E_{n}\right\}$ is the spectrum of $-\Delta$ [in the presence of a magnetic field, $\left.\Delta \rightarrow(\nabla-2 \mathrm{i} e A)^{2}\right]$. The interest in introducing $S(\gamma)$ is that it can be related to the determinant of a $V \times V$-matrix, where $V$ is the number of vertices, that encodes all information on the network (topology, length of the wires, magnetic field, connection to reservoirs). We label vertices by greek letters. $l_{\alpha \beta}$ designates the length of the wire $(\alpha \beta)$ and $\theta_{\alpha \beta}$ the circulation of the vector potential along the wire. The topology is encoded in the adjacency matrix : $a_{\alpha \beta}=1$ if $\alpha$ and $\beta$ are linked by a wire, $a_{\alpha \beta}=0$ otherwise. $\lambda_{\alpha}=\infty$ if $\alpha$ is connected to a reservoir and $\lambda_{\alpha}=0$ if not. We introduce the matrix

$$
\begin{array}{r}
\mathcal{M}_{\alpha \beta}=\delta_{\alpha \beta}\left(\lambda_{\alpha}+\sqrt{\gamma} \sum_{\mu} a_{\alpha \mu} \operatorname{coth} \sqrt{\gamma} l_{\alpha \mu}\right) \\
-a_{\alpha \beta} \sqrt{\gamma} \frac{\mathrm{e}^{-\mathrm{i} \theta_{\alpha \beta}}}{\sinh \sqrt{\gamma} l_{\alpha \beta}}
\end{array}
$$

where the $a_{\alpha \mu}$ constraints the sum to run over neighbouring vertices. Then 24

$$
S(\gamma)=\prod_{(\alpha \beta)} \frac{\sinh \sqrt{\gamma} l_{\alpha \beta}}{\sqrt{\gamma}} \operatorname{det} \mathcal{M}
$$

where the product runs over all wires. We now consider a large square network of size $N_{x} \times N_{y}$ made of wires of length $l_{\alpha \beta}=a \forall(\alpha \beta)$. For simplicity we impose periodic boundary conditions (topology of a torus), which is inessential as soon as the total size of the network remains small compared to $L_{\varphi}$. At zero magnetic field the spectrum of the adjacency matrix is $\epsilon_{n, m}=2 \cos \left(2 n \pi / N_{x}\right)+$ $2 \cos \left(2 m \pi / N_{y}\right)$, with $n=1, \cdots, N_{x}$ and $m=$ $1, \cdots, N_{y}$. Therefore

$$
\begin{aligned}
& S(\gamma)=\left(2 \frac{\sinh \sqrt{\gamma} a}{\sqrt{\gamma}}\right)^{N_{x} N_{y}} \\
& \times \prod_{n=1}^{N_{x}} \prod_{m=1}^{N_{y}}\left(2 \cosh \sqrt{\gamma} a-\cos \frac{2 \pi n}{N_{x}}-\cos \frac{2 \pi m}{N_{y}}\right)
\end{aligned}
$$

FIG. 1: $\Delta \sigma_{\mathrm{WL}}$ in unit of $2 e^{2} / h$ as a function of $L_{\varphi} / a$ (at zero magnetic field). The dashed line is the $1 d$ result. The dotted line is the 2d limit eq. (10).

1d limit. - In the limit $L_{\varphi} \ll a($ i.e. $\sqrt{\gamma} a \gg 1)$ :

$$
\Delta \sigma_{\mathrm{WL}}=-\frac{2 e^{2}}{h}\left(L_{\varphi}-\frac{L_{\varphi}^{2}}{2 a}+O\left(\mathrm{e}^{-2 a / L_{\varphi}}\right)\right)
$$

We compare with the result for a wire of length $a$ connected at its extremities : $\Delta \sigma_{\mathrm{WL}}^{\mathrm{wire}} \simeq-\frac{2 e^{2}}{h}\left(L_{\varphi}-\right.$ $\left.\frac{L_{\varphi}^{2}}{a}\right)$. As we can see the dominant terms coincide. Deviations appear when $L_{\varphi} / a$ increases since trajectories begin to feel the topology of the network. This is already visible by comparing the second terms of the expansions.

2d limit. - In the limit $L_{\varphi} \gg a($ i.e. $\sqrt{\gamma} a \ll 1)$, we 
obtain

$$
\begin{array}{r}
\frac{1}{\mathrm{Vol}} \frac{\partial}{\partial \gamma} \ln S(\gamma)=\frac{a}{2 \pi}\left[\ln \left(4 L_{\varphi} / a\right)+\frac{\pi}{6}\right. \\
\left.+O\left(\frac{a^{2}}{L_{\varphi}^{2}} \ln \frac{L_{\varphi}}{a}\right)\right]
\end{array}
$$

The conductivity reads

$$
\Delta \sigma_{\mathrm{WL}} \simeq-\frac{2 e^{2}}{h} a\left[\frac{1}{\pi} \ln \left(L_{\varphi} / a\right)+C_{\mathrm{WL}}\right]
$$

with $C_{\mathrm{WL}}=\frac{2 \ln 2}{\pi}+\frac{1}{6} \simeq 0.608$. As noticed in the beginning of the section, eqs. 810 should be divided by the cross-section $s$ of the wires. In the $2 \mathrm{~d}$ limit, diffusive trajectories expand over distances larger than $a$ and feel the two dimensional nature of the system, being the reason why (10) is reminiscent of the $2 \mathrm{~d}$ result. It is interesting to point that the network provides a natural cutoff (the length of the wires, $a$ ) while the computation of the WL for a plane in the diffusion approximation requires to introduce a cutoff by hand for lower times in eq. (1), which is the elastic scattering time $\tau_{e}$. In this latter case the constant added to the logarithmic behaviour is not well controlled since it depends on the cutoff procedure (the computation of the constant for a plane requires to go beyond the diffusion approximation and leads to 31 $-\frac{e^{2}}{\pi h} \ln \left(2 L_{\varphi}^{2} / \ell_{e}^{2}+1\right) \simeq-\frac{2 e^{2}}{h}\left[\frac{1}{\pi} \ln \left(L_{\varphi} / \ell_{e}\right)+\frac{1}{2 \pi} \ln 2\right]$ since $\left.\ell_{e} \ll L_{\varphi}\right)$.

\section{AL'TSHULER-ARONOV CORRECTION}

At first order in the electron-electron interaction, the exchange term is the dominant contribution to the correction to the conductivity ${ }^{8,10,11,32}$

$$
\begin{aligned}
\Delta \sigma_{\mathrm{ee}}= & -\frac{2 \sigma_{0}}{d \pi \mathrm{Vol}} \int_{-\infty}^{+\infty} \mathrm{d} \omega \frac{\partial}{\partial \omega}\left(\omega \operatorname{coth} \frac{\omega}{2 T}\right) \\
& \times \operatorname{Im} \sum_{\vec{q}} D \vec{q}^{2} \frac{U(\vec{q}, \omega)}{\left(-\mathrm{i} \omega+D \vec{q}^{2}\right)^{3}}
\end{aligned}
$$

where $U(\vec{q}, \omega)$ is the dynamically screened interaction. Within the RPA approximation and in the small $\vec{q}$ and $\omega$ limit, the interaction takes the form ${ }^{33} U(\vec{q}, \omega) \simeq \frac{1}{2 \rho_{0}} \frac{-i \omega+D \vec{q}^{2}}{D \vec{q}^{2}}$ where $\rho_{0}$ is the density of states per spin channel. Replacing the Drude conductivity by its expression $\sigma_{0}=2 e^{2} \rho_{0} D$ and performing an integration by parts, we get

$$
\begin{aligned}
\Delta \sigma_{\mathrm{ee}}= & -\frac{2 e^{2} D}{\pi d \mathrm{Vol}} \int \mathrm{d} \omega \frac{\partial^{2}}{\partial \omega^{2}}\left(\omega \operatorname{coth} \frac{\omega}{2 T}\right) \\
& \times \operatorname{Re} \sum_{\vec{q}} \frac{1}{-\mathrm{i} \omega+D \vec{q}^{2}}
\end{aligned}
$$

After Fourier transform, the result can be cast in the form 11 :

$$
\Delta \sigma_{\mathrm{ee}}=-\lambda_{\sigma} \frac{e^{2} D}{\pi} \int_{0}^{\infty} \mathrm{d} t\left(\frac{\pi T t}{\sinh \pi T t}\right)^{2} \mathcal{P}_{d}(t)
$$

For the exchange term considered here, one finds $\lambda_{\sigma}=4 / d$. Further calculation yields $\lambda_{\sigma} \simeq$ $\frac{4}{d}-\frac{3}{2} F$, where $F$ is the average of the interaction on the Fermi surface (see definition in Refs,,$\underline{8,9}$ ). This expression of $\lambda_{\sigma}$ is valid in the perturbative regime, $F \ll 1$; nonperturbative expression is given in Refs $6,7,8,9$. $\mathcal{P}_{d}(t)$ is the space integrated return probability $\mathcal{P}_{d}(t)=\int \frac{\mathrm{d} x}{\mathrm{Vol}_{\mathrm{ol}}} \mathcal{P}_{d}(x, x ; t)$, where $\mathcal{P}_{d}\left(x, x^{\prime} ; t\right)$ is solution of a classical diffusion equation similar to the equation for $\mathcal{P}_{c}\left(x, x^{\prime} ; t\right)$, apart that it does not feel the magnetic field : $\left[\partial_{t}-D \Delta\right] \mathcal{P}_{d}\left(x, x^{\prime} ; t\right)=\delta\left(x-x^{\prime}\right) \delta(t)$. Therefore the Laplace transform of $\mathcal{P}_{d}(t)$ is given by $\partial_{\gamma} \ln S(\gamma)$ with $\theta_{\alpha \beta}=0$. It is interesting to point out that (13) has a similar structure to (1) with a different cutoff procedure for large time. It also involves a different scale : the temperature dependence of $\Delta \sigma_{\mathrm{ee}}$ is driven by the length scale $L_{T}$ instead of $L_{\varphi}$ for the weak-localization correction $\Delta \sigma_{\mathrm{WL}}$.

Up to eq. (13) the discussion is rather general and nothing has been specified on the system. We have seen in section II that the WL for the square network presents a dimensional crossover from $1 \mathrm{~d}$ to $2 \mathrm{~d}$ by tuning $L_{\varphi} / a$. A similar dimensional crossover occurs for the AA correction by tuning $L_{T} / a$ as we will see. This remark raises the question of the dimension $d$ in eq. (11). To answer this question we should return to the origin of the factor $1 / d$ : the current lines in the conductivity $\sigma_{i j}$ produce a factor $q_{i} q_{j}$ replaced by $\delta_{i j} \frac{1}{d} \vec{q}^{2}$ after angular integration. Since in a network the diffusion in the wires has a 1d structure (provided that $W \ll L_{T} \sim \sqrt{D / \omega}$, where $W$ is the width of the wires), the dimension in $\lambda_{\sigma}$ is $d=1$. Therefore we have for the network $\lambda_{\sigma}^{\text {network }} \simeq 4-\frac{3}{2} F$.

If one now expands the thermal function in (13) as :

$$
\left(\frac{y}{\sinh y}\right)^{2}=4 y^{2} \sum_{n=1}^{\infty} n \mathrm{e}^{-2 n y}
$$

we can also relate $\Delta \sigma_{\text {ee }}$ to the spectral determinant. We obtain :

$$
\Delta \sigma_{\mathrm{ee}}=-\lambda_{\sigma} \frac{e^{2}}{\pi \mathrm{Vol}} \sum_{n=1}^{\infty} \frac{1}{n}\left[\gamma^{2} \frac{\partial^{3}}{\partial \gamma^{3}} \ln S(\gamma)\right]_{\gamma=\frac{2 n \pi}{L_{T}^{2}}}
$$

which is the central result of this paper. It is the starting point of the discussion below.

Application to the case of the square network.-We have to compute $\gamma^{2} \frac{\partial^{3}}{\partial \gamma^{3}} \ln S(\gamma)$. We start from (77) and compute its second derivative. We obtain after 
some algebra :

where the function $\varphi(x)$ is given by:

$$
\Delta \sigma_{\mathrm{ee}}=-\lambda_{\sigma} \frac{e^{2}}{h} \frac{a}{8} \sum_{n=1}^{\infty} \frac{1}{n} \varphi\left(\sqrt{2 n \pi} \frac{a}{L_{T}}\right)
$$

$$
\begin{aligned}
\varphi(x) & =-\frac{8}{x^{2}}+\frac{2 x \cosh x}{\sinh ^{3} x}+\frac{3}{\sinh ^{2} x}+\frac{3 \operatorname{coth} x}{x} \\
& +\frac{2}{\pi}\left\{\left[\frac{3 \tanh x}{x}-3\right] \mathrm{K}\left(\frac{1}{\cosh x}\right)+\left[3-\frac{2 x}{\sinh 2 x}\right] \mathrm{E}\left(\frac{1}{\cosh x}\right)\right\},
\end{aligned}
$$

$\mathrm{E}(x)$ being the complete elliptic integral of second kind ${ }^{30}$. The function $\varphi(x)$ is plotted in figure 2 and its limiting behaviours are easily obtained ${ }^{30}$ :

$$
\begin{aligned}
\varphi(x) & =\frac{4}{\pi}+O\left(x^{2}\right) & \text { for } x \rightarrow 0 \\
& =\frac{6}{x}-\frac{8}{x^{2}}+O\left(x \mathrm{e}^{-2 x}\right) & \text { for } x \rightarrow \infty
\end{aligned}
$$

The $L_{T}$ dependence of AA correction on a square network is displayed on figure 3, where we have plotted $\Delta \sigma_{\text {ee }}\left(L_{T}\right)$ given by eq. (16). The dimensional crossover now occurs by tuning the ratio $L_{T} / a$. We consider the two limits.

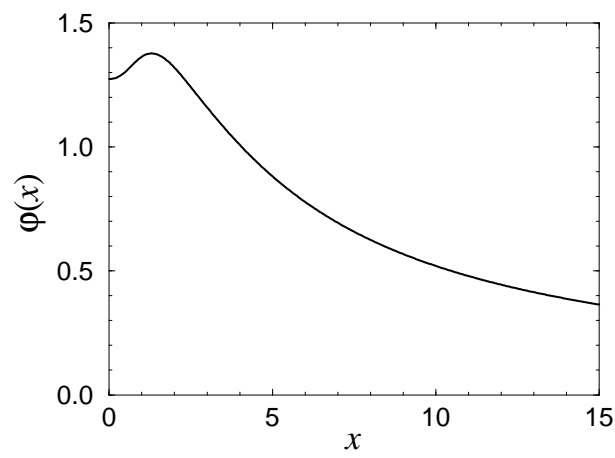

FIG. 2: The function $\varphi(x)$ of eq. 177).

1d limit.- For $L_{T} \ll a$ we can replace the expansion (19) in the series (16). Therefore

$$
\Delta \sigma_{\mathrm{ee}} \simeq-\lambda_{\sigma} \frac{e^{2}}{h}\left(\frac{3 \zeta(3 / 2)}{4 \sqrt{2 \pi}} L_{T}-\frac{\pi}{12} \frac{L_{T}^{2}}{a}\right)
$$

with $\frac{3 \zeta(3 / 2)}{4 \sqrt{2 \pi}} \simeq 0.782$. The dominant term again coincides with the one for a connected wire $8,10,11$ while the second differs by a factor 2 , as for the WL [see discussion after eq. (8)].

$2 d$ limit.- In the limit $L_{T} \gg a$ we introduce $\mathcal{N}=\left(L_{T} / a\right)^{2}$ and cut the sum (16) in two pieces: $\sum_{1}^{\infty}=\sum_{1}^{\mathcal{N}}+\sum_{\mathcal{N}}^{\infty}$. It is clear from the limit be-

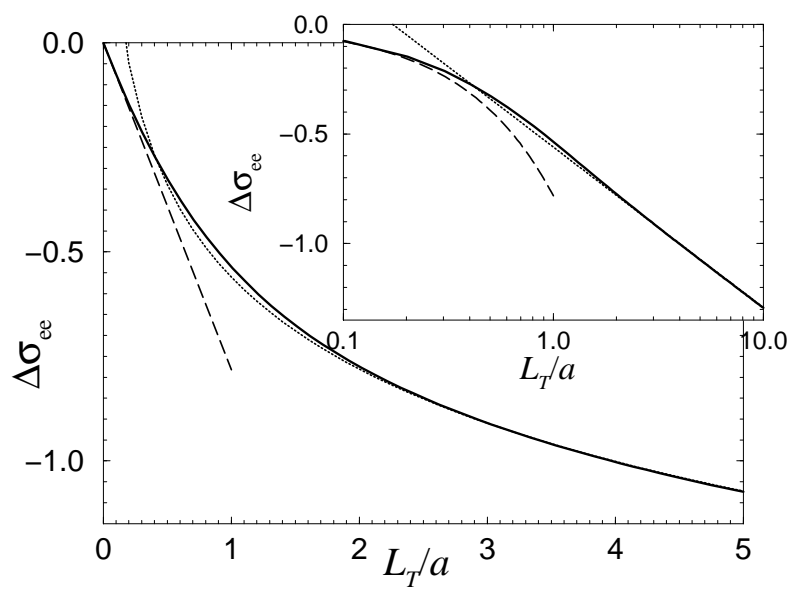

FIG. 3: The continuous line is $\Delta \sigma_{\mathrm{ee}}$ in unit of $\lambda_{\sigma} \frac{e^{2}}{h}$ as a function of $L_{T} / a$ (the series (16) is computed numerically). The dashed line is the $1 d$ limit, eq. (20), and the dotted curve is the 2d limit, eq. 21).

haviours of $\varphi(x)$ that the first sum diverges logarithmically with $\mathcal{N}$ while the second brings a negligible contribution of order $\mathcal{N}^{0}$. Therefore :

$$
\Delta \sigma_{\mathrm{ee}} \simeq-\lambda_{\sigma} \frac{e^{2}}{h} a\left[\frac{1}{\pi} \ln \left(L_{T} / a\right)+C_{\mathrm{ee}}\right]
$$

The constant is estimated numerically. We find $C_{\text {ee }} \simeq 0.56$.

The two eqs. (20|21) should be divided by the cross-section $s$ of the wires.

The two functions $\Delta \sigma_{\mathrm{WL}}\left(\mathcal{B}=0, L_{\varphi}\right)$ (figure 1) and $\Delta \sigma_{\text {ee }}\left(L_{T}\right)$ (figure 3) are very similar. Apart from the prefactors $2 e^{2} / h$ and $\lambda_{\sigma} e^{2} / h$ which account respectively for the spin degeneracy and the interaction strength, the linear behaviours at the origin have a different slope (1 and 0.782 ) and the logarithmic behaviours are slightly shifted : $C_{\mathrm{WL}} \simeq 0.61$ and $C_{\mathrm{ee}} \simeq 0.56$. 


\section{COMPARISON WITH EXPERIMENTS}

The AA correction has been recently measured by Mallet et a $\underline{34}$ in networks of silver wires with $310^{4}$ and $10^{5}$ cells, lattice spacing $a=0.64 \mu \mathrm{m}$ and diffusion constant $D \simeq 100 \mathrm{~cm}^{2} / \mathrm{s}$. The diffusion constant $D$ has been measured separately (through measurement of the Drude conductance), therefore we can compare our result (16) with experiment using one fitting parameter only : the interaction parameter $\lambda_{\sigma}$. The 2d logarithmic behaviour (21) has been observed in the range $100 \mathrm{mK}<T<1 \mathrm{~K}$ from which the value $\lambda_{\sigma}^{\exp } \simeq 3.1$ was extracted, in agreement with similar measurements performed on a long silver wire for which $^{34,35} \lambda_{\sigma}^{\text {exp}}$, wire $\simeq 3.2$. We now compare with the theoretical value : for silver Fermi wavelength is $k_{F}^{-1}=0.083 \mathrm{~nm}$ and Thomas-Fermi screening length $\kappa^{-1}=1 / \sqrt{8 \pi \rho_{0} e^{2}}=0.055 \mathrm{~nm}$. In the Thomas-Fermi approximation, the parameter $F$ is given by $11=\left(\frac{\kappa}{2 k_{F}}\right)^{2} \ln \left[1+\left(\frac{2 k_{F}}{\kappa}\right)^{2}\right]$, therefore $F \simeq 0.58$. Using the $1 \mathrm{~d}$ nonperturbative expres$\operatorname{sion}^{8} \lambda_{\sigma}=4+\frac{48}{F}(\sqrt{1+F / 2}-1-F / 4)$, we get $\lambda_{\sigma}^{\text {th }} \simeq 3.24$, close to the experimental value.

\section{CONCLUSION}

Equations (1516) are our main results. The first one shows that AA and WL can be formally related :

$$
\begin{aligned}
\Delta \sigma_{\mathrm{ee}}\left(L_{T}\right) & =\frac{\lambda_{\sigma}}{2} \\
\times & \sum_{n=1}^{\infty} \frac{1}{n}\left[\gamma^{2} \frac{\partial^{2}}{\partial \gamma^{2}} \Delta \sigma_{\mathrm{WL}}\left(L_{\varphi}\right)\right]_{\gamma \equiv \frac{1}{L_{\varphi}^{2}}=\frac{2 n \pi}{L_{T}^{2}}}
\end{aligned}
$$

The validity of this relation is the same as for eqs. (12) : the system should be such that it is meaningful to average uniformly the nonlocal conductivity $\sigma\left(r, r^{\prime}\right)$ to get the local conductivity $\sigma=\int \frac{\mathrm{d} r \mathrm{~d} r^{\prime}}{\mathrm{Vol}} \sigma\left(r, r^{\prime}\right)$. A similar discussion has been proposed to relate WL and conductivity fluctuations (see appendix E of Ref. ${ }^{29}$ ).

Our starting point (11) is a formulation in the Fourier space, what implicitly assumes translation invariance. Whereas this assumption seems reasonable for a large regular network such as the square network studied in this article, its validity is not clear for networks of arbitrary topology, what would need further developments.

We have computed the AA correction in a large square network and shown that the result interpolates between the $1 \mathrm{~d}$, eq. (20), and a $2 \mathrm{~d}$ result, eq. (21). Interestingly, the $2 \mathrm{~d}$ limit in a network involves a $1 \mathrm{~d}$ constant $\lambda_{\sigma}^{\text {network }} \simeq 4-\frac{3}{2} F$, what is confirmed by experiments, as discussed in section IV

The interest of the network compared to the plane is to control the constant $C_{\mathrm{ee}}$ of eq. (21) : for a plane, a cutoff must be introduced in eq. (13) at short time $t \sim \tau_{e}$ and the constant $C_{\mathrm{ee}}$ is replaced by a number that depends on the precise cutoff procedure. Experimentally, it would be interesting to observe the crossover from (20) to (21) by varying $L_{T} / a$. This was not possible in experiments of Mallet et a $\underline{\underline{34}}^{34}$ described in section [V] because measurements are complicated by the fact that electron-phonon interaction also brings a temperature-dependent contribution, $\Delta \sigma_{\mathrm{e}-\mathrm{ph}}$, at high temperature (above few Kelvins). The conductivity is given by $\sigma=\sigma_{0}+\Delta \sigma_{\mathrm{WL}}+$ $\Delta \sigma_{\mathrm{ee}}+\Delta \sigma_{\mathrm{e}-\mathrm{ph}}$. The WL can be suppressed by a magnetic field however the electron-phonon contribution is difficult to separate from $\Delta \sigma_{\mathrm{ee}}$. Therefore the network should be patterned in a way such that the crossover $1 \mathrm{~d}-2 \mathrm{~d}$ remains below $T \sim 1 \mathrm{~K}$ where $\Delta \sigma_{\mathrm{e}-\mathrm{ph}}$ is negligible. As an example we consider the silver networks studied in Ref ${ }^{21}$ for which $L_{T}=0.27 \times T^{-1 / 2}\left(L_{T}\right.$ in $\mu \mathrm{m}$ and $T$ in $\left.\mathrm{K}\right)$. In order to see clearly the $1 \mathrm{~d}$ and the $2 \mathrm{~d}$ regimes it would be convenient to study two networks with different lattice spacings. If temperature is constrained by $10 \mathrm{mK}<T<1 \mathrm{~K}$, for $a=0.5 \mu \mathrm{m}$ we have $0.5 \lesssim L_{T} / a \lesssim 5$, which probes the $2 \mathrm{~d}$ regime over one decade. A second lattice with $a \sim 5 \mu \mathrm{m}$ would allow to probe the $1 \mathrm{~d}$ regime since in this case $0.05 \lesssim L_{T} / a \lesssim 0.5$.

\section{Acknowledgements}

We have benefitted from stimulating discussions with Christopher Bäuerle, Hélène Bouchiat, Meydi Ferrier, François Mallet, Laurent Saminadayar and Félicien Schopfer.
1 B. L. Altshuler, A. G. Aronov, and D. E. Khmelnitsky, Effects of electron-electron collisions with small energy transfers on quantum localisation, J. Phys. C: Solid St. Phys. 15, 7367 (1982).

2 S. Chakravarty and A. Schmid, Weak localization: the quasiclassical theory of electrons in a random potential, Phys. Rep. 140(4), 193 (1986).
3 B. L. Al'tshuler and A. G. Aronov, Contribution to the theory of disordered metals in strongly doped semiconductors, Sov. Phys. JETP 50(5), 968 (1979).

4 B. L. Al'tshuler, D. E. Khmel'nitzkiŭ, A. I. Larkin, and P. A. Lee, Magnetoresistance and Hall effect in a disordered two-dimensional electron gas, Phys. 
Rev. B 22(11), 5142 (1980).

5 B. L. Altshuler and A. G. Aronov, Fermi-liquid theory of the electron-electron interaction effects in disordered metals, Solid State Commun. 46, 429 (1983).

6 A. M. Finkel'shteln, Influence of Coulomb interaction on the properties of disordered metals, Sov. Phys. JETP 57(1), 97 (1983).

7 C. Castellani, C. Di Castro, P. A. Lee, and M. Ma, Interaction-driven metal-insulator transitions in disordered fermion systems, Phys. Rev. B 30(2), 527 (1984).

8 B. L. Altshuler and A. G. Aronov, Electron-electron interaction in disordered conductors, in Electronelectron interactions in disordered systems, edited by A. L. Efros and M. Pollak, page 1, NorthHolland, 1985.

9 P. A. Lee and T. V. Ramakrishnan, Disordered electronic systems, Rev. Mod. Phys. 57, 287 (1985).

10 I. L. Aleiner, B. L. Altshuler, and M. E. Gershenson, Interaction effects and phase relaxation in disordered systems, Waves Random Media 9, 201 (1999).

11 É. Akkermans and G. Montambaux, Mesoscopic physics of electrons and photons, Cambridge University Press, 2007.

12 It is worth mentioning that a similar effect exists at higher temperature, in the ballistic regime $\left(\tau_{\text {ee }} \ll\right.$ $\left.\tau_{e}\right)$; Ref ${ }^{13}$ provides a nice review on this point and describes the crossover between the two regimes.

${ }^{13}$ G. Zala, B. N. Narozhny, and I. L. Aleiner, Interaction corrections at intermediate temperatures: Longitudinal conductivity and kinetic equation, Phys. Rev. B 64, 214204 (2001).

14 A. E. White, M. Tinkham, W. J. Skocpol, and D. C. Flanders, Evidence for interaction effects in the low-temperature resistance rise in ultrathin metallic wires, Phys. Rev. Lett. 48(25), 1752 (1982).

15 P. M. Echternach, M. E. Gershenson, H. M. Bozler, A. L. Bogdanov, and B. Nilsson, Temperature dependence of the resistance of one-dimensional metal films with dominant Nyquist phase breaking, Phys. Rev. B 50(8), 5748 (1994).

16 F. Pierre, A. B. Gougam, A. Anthore, H. Pothier, D. Esteve, and N. O. Birge, Dephasing of electrons in mesoscopic metal wires, Phys. Rev. B 68, 085413 (2003).

17 C. Bäuerle, F. Mallet, F. Schopfer, D. Mailly, G. Eska, and L. Saminadayar, Experimental Test of the Numerical Renormalization Group Theory for Inelastic Scattering from Magnetic Impurities, Phys. Rev. Lett. 95, 266805 (2005).

18 B. Pannetier, J. Chaussy, R. Rammal, and P. Gandit, First Observation of Altshuler-Aronov-Spivak effect in gold and copper, Phys. Rev. B 31(5), 3209
(1985).

19 G. J. Dolan, J. C. Licini, and D. J. Bishop, Quantum Interference Effects in Lithium Ring Arrays, Phys. Rev. Lett. 56(14), 1493 (1986).

20 M. Ferrier, L. Angers, A. C. H. Rowe, S. Guéron, H. Bouchiat, C. Texier, G. Montambaux, and D. Mailly, Direct measurement of the phase coherence length in a GaAs/GaAlAs square network, Phys. Rev. Lett. 93, 246804 (2004).

21 F. Schopfer, F. Mallet, D. Mailly, C. Texier, G. Montambaux, L. Saminadayar, and C. Bäuerle, Dimensional crossover in quantum networks: from mesoscopic to macroscopic physics, Phys. Rev. Lett. 98, 026807 (2007).

22 B. Douçot and R. Rammal, Quantum oscillations in normal-metal networks, Phys. Rev. Lett. 55(10), 1148 (1985).

23 B. Douçot and R. Rammal, Interference effects and magnetoresistance oscillations in normal-metal networks: 1. weak localization approach, J. Physique 47, 973-999 (1986).

24 M. Pascaud and G. Montambaux, Persistent currents on networks, Phys. Rev. Lett. 82, 4512 (1999).

25 Pascaud \& Montambaux have rather considered thermodynamic properties. The nonlocal effects in networks have been further investigated in Ref. ${ }^{27}$.

26 E. Akkermans, A. Comtet, J. Desbois, G. Montambaux, and C. Texier, On the spectral determinant of quantum graphs, Ann. Phys. (N.Y.) 284, 10-51 (2000).

27 C. Texier and G. Montambaux, Weak localization in multiterminal networks of diffusive wires, Phys. Rev. Lett. 92, 186801 (2004).

${ }^{28}$ T. Ludwig and A. D. Mirlin, Interaction-induced dephasing of Aharonov-Bohm oscillations, Phys. Rev. B 69, 193306 (2004).

29 C. Texier and G. Montambaux, Dephasing due to electron-electron interaction in a diffusive ring, Phys. Rev. B 72, 115327 (2005).

30 I. S. Gradshteyn and I. M. Ryzhik, Table of integrals, series and products, Academic Press, fifth edition, 1994.

31 A. Cassam-Chenai and B. Shapiro, Two dimensional weak localization beyond the diffusion approximation, J. Phys. I France 4, 1527 (1994).

32 The formula (5.1) of Ref. $\frac{8}{-}$ has the wrong sign.

33 This interaction assumes that the screening length is smaller than the transverse size of the wire.

34 F. Mallet et al, to be published (2007).

35 L. Saminadayar, P. Mohanty, R. A. Webb, P. Degiovanni and C. Bäuerle, Phase coherence in the presence of magnetic impurities, Physica E, to be published (2007). 